\title{
Residual Cancer Burden Class
}

National Cancer Institute

\section{Source}

National Cancer Institute. Residual Cancer Burden Class. NCI Thesaurus. Code C160724.

Residual cancer burden classes that use pre-defined cut-points representing prog ressively greater extents of residual cancer and indicating a greater risk of recurrence. 